\title{
Cognitive Process Visibility: An Embedded Process Monitoring Approach in an Intelligent Learning Module
}

\author{
Adetokunbo A. Adenowo \\ Dept. of Electronic \& Computer Engineering, Faculty of Engineering, Lagos State University, Lagos - Nigeria. \\ adetokyom@yahoo.com
}

Received: $14^{\text {th }}$ February 2018

Accepted: $26^{\text {th }}$ July 2018

Published: $20^{\text {th }}$ September 2018

https://doi.org/10.47545/etrj.2018.3.2.041

\begin{abstract}
Theoretically, the visibility of the cognitive process of a learner tends to enhance knowledge gap identification, tutoring guidance provision and learning process adaptation in order to achieve effective learning. Towards the aforementioned, what approach would reveal the cognitive process of a learner during a learning process? Thus, this work presents an interface-based approach that is underpinned by the concept of "embedded Process Monitoring" (ePM). The conception/deployment of ePM aims to make visible the cognitive process of learners. This paper examines whether the proposed interface-driven ePM approach achieves the design/research objectives. Accordingly, a prototype software learning platform termed intelligent learning module was designed and subjected to evaluation within a numerical problem-solving context. Human subjects were sampled using a questionnaire. Quantitative data from the questionnaire-based evaluation was statistically analysed, while the subjective aspects were subjected to thematic analysis. Results indicate that the interfacebased ePM reveals the cognitive process of learners; aid the generation of timely and relevant feedbacks. Also, there were few indications that the interface-driven ePM approach obstructs learning. Notwithstanding, the prototype learning platform provides an alternative route(s) that enable(s) advanced learners to construct knowledge.
\end{abstract}

Keywords: Cognitive Process, Diagnostic Feedback, Intelligent Learning Environment, Intelligent Tutoring System, Process Monitoring.

\section{INTRODUCTION}

This work proposes a design that is based on the concept of embedded process monitoring (ePM). ePM is a tutoring concept or strategy that is operationalized through an interface. The concept/strategy attempts to track/make visible the cognitive process of a learner during a learning process, thus enable provision of appropriate learning implements the ePM concept. It's designed to provide intelligently guided problem-solving experience. This design approach attempts to extend the research focus of the field of intelligent tutoring system (ITS).guidance with the overall goal of achieving effective learning. To illustrate the concept, this work presents a prototype software learning platform, termed Intelligent Learning Module (iLM), which implements the ePM concept. It's designed to provide intelligently guided problem-solving experience. This design approach attempts to extend the research focus of the field of intelligent tutoring system (ITS).

Generally, ITSs are computer-based learning systems that can adapt, recommend and deploy personalised domain contents, teach and help students work with problems [1-4]. ITS can be pedagogy and/or performance oriented tool [5]. Pedagogy-oriented ITSs provide instruction or course-like attributes with focus on how to sequence and teach canned domain content. On the other hand, performance-oriented ITSs provide rich learning environments that support learning by practice and receive feedbacks [5-6]. However, current work is domiciled within the performance category of ITS. Thus, the prototype learning platform (i.e. iLM) is designed to support learning by practice and feedbacks through built-in interface-driven ePM features that provide required intelligence. The interface-driven ePM concept as implemented in the iLM targets the following research objectives, viz:: [i] investigate if ePM increases visibility of the cognitive process of target users of the learning platform; and [ii] determine the perception of target users regarding the impact of ePM on the feedback generation. 
A review of literatures within the general field of intelligent tutoring system (including adaptive learning systems, collaborative learning platforms, etc.) indicates that most research efforts deploy artificial intelligence (AI) techniques. The utilisation of AI techniques aimed to model, as well as provides requisites intelligence for the components of the learning systems within the aforementioned field(s). The learning systems' components modelled include: students' learning behaviour, teaching strategies and domain knowledge. For instance, Bouchet et al. [7] used a cluster algorithm to explore data collected while learning circulatory system with MetaTutor, an agent-based ITS designed to foster self-regulated learning; Crockett et al. [1] utilised Fuzzy decision trees to model learners' behaviour and predict their respective learning styles; Hooshyar et al. [8-9] utilised Bayesian networks to implement decision making in order to aid students problem solving activities.

Similarly, Chieu et al. [10] integrated temporal Bayesian networks and fine-grained didactic analysis to model and diagnose students during their learning process. Piech, et al. [11] deployed Recurrent Neural Networks (RNN) to model student learning behaviour; Ostrow, et al. [12] provided intelligent learning guidance through the tabling of algorithmically determined partial credit score and problem difficulty per student's problem state; and Eagle, et al. [13] considered how to individualise Bayesian knowledge tracing (BKT) parameters to model learners. Also, Han [14] designed a teaching model that is based on domain ontology; the latter evaluates the ontology to improve the cognitive activity of college students as well as, help teachers determine learners' situation and adapt teaching strategy.

In spite the aforementioned research papers, and others not cited, accomplished some levels of successes (as recorded in their respective literatures), learning systems so presented are majorly AI-based systems. These AIbased systems are still considered to be complex, hard and time-consuming to design/build based on several factors including its architecture [15]. Also, none of the above mentioned research works considered the design of an intelligent/adaptive learning system that addressed visibility of cognitive process in order to aid learning process, or at least, from a non-AI approach.

It's on this background that current work explores an entire different path from the traditional AI techniques. This paper considers a design approach to actualise intelligence in a learning system from the angle of humancomputer interaction. That is, it proposes interface-driven ePM concept to model the teaching and student components of an intelligent learning platform. Specifically, the proposed interface-based ePM learning platform aims: to model students' learning behaviours; track/make visible their cognitive process in order to provide required guidance through automated generation of timely and relevant diagnostic feedbacks during learning-by-practise session(s). Thus, the ePM implementation intends to bring to the surface (or make visible) the cognitive process taking place in the cognitive system of a learner that is attempting to solve numerical problem(s) on a learning platform.

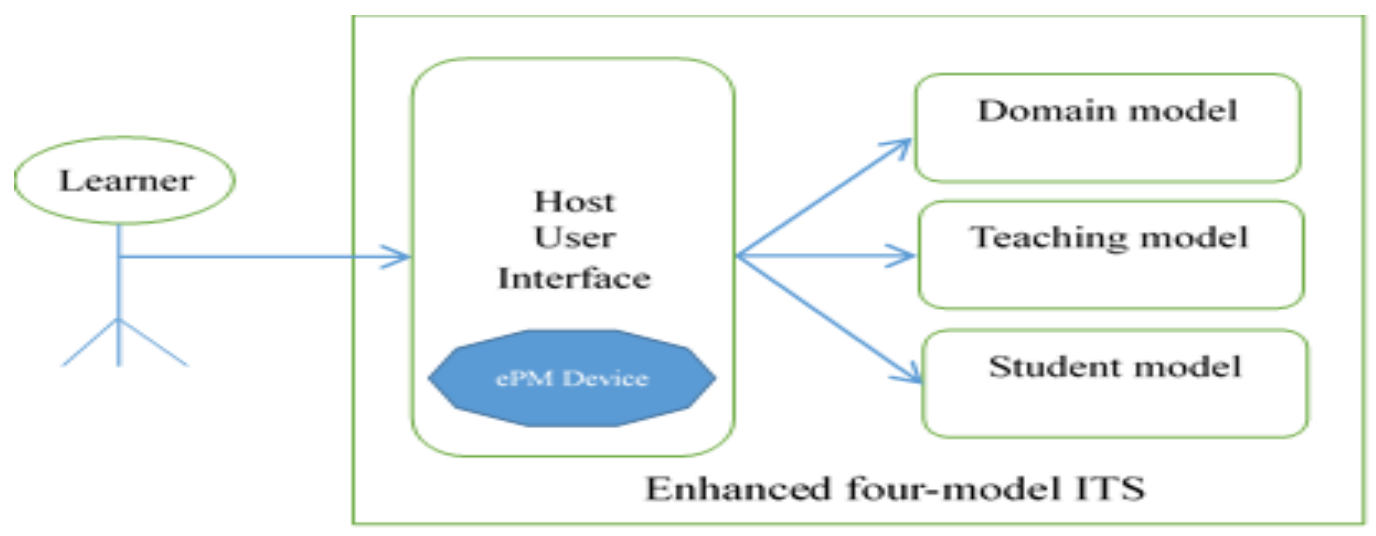

Fig. 1: Enhanced four-model intelligent learning module architecture

Although, ePM concept is designed into the iLM in this research, the latter still draws structure from established ITS architecture. Structurally, previous works propounded modular ITS architectures. Murray [5], for instance, argued a two-model architecture consisting of: (i) what to teach-that is, the subject-matter or content such as mathematics, physics, algebra, programming, etc.; (ii) how to teach-that is, teaching strategies such as one-onone tutoring, coaching, simulation, etc. In same light, [4] provided insight into two additional models namely: 
(a) student model-inference on student's mastery of topics or tasks towards dynamic adaption of subject matter; and (b) a user interface-medium through which a student/user interacts with the learning environment. Thus, a generalised four-model architecture (consisting of: domain, tutoring, student and interface modules) has been established in the ITS literatures [4]. Flowing from the foregoing, current work structurally considers enhanced four-model architecture; a structure that hosts a virtual device within its user interface component in order to drive the proposed ePM concept (see fig. 1 above).

\section{MATERIALS AND METHOD}

\subsection{Research Process Phases}

A three-stage research process was implemented. In the first phase, traditional review approach was undertaken. Literatures searched were based on themes, topics and/or keywords related to current research topic/objectives. Attention was given to literatures on students' learning behaviour/modelling, teaching strategies, user interface design and domain knowledge representation in a technology platform (such as intelligent tutoring system).

Second phase relates to the authoring of a prototype iLM for a chosen/target domain knowledge using an existing authoring tool (ILABS-Intelligent Learning Activity Builder System). The prototype iLM provides basis to operationalize and evaluate the ePM concept. The prototype iLM can be launched either: within its authoring tool (as shown in fig. 2); or by downloading the iLM from a customised repository (that contains priori-generated iLMs), then deployed within a learning management system.

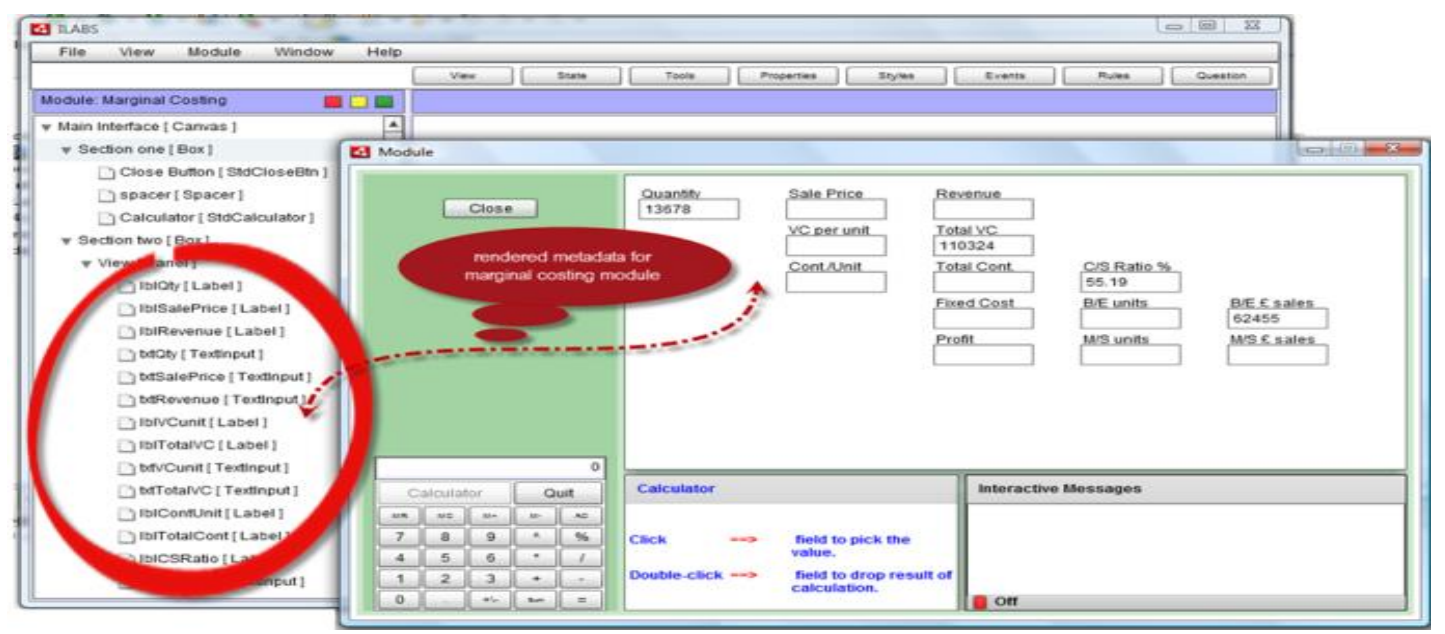

Fig. 2: Prototype intelligent learning module generated and lunched within an existing authoring tool.

The third phase deals with the evaluation of the interface-driven ePM-based intelligent learning module. The module was deployed in real educational settings involving two institutions of learning. A sample of three hundred (300) students was taken. A purposely designed questionnaire was deployed since no standard instrument exist (as argued in [16]); pretested to determine whether it measures what it is meant to measure, ensure its quality and efficiency (see [17, 18]). The questionnaire instrument consists of open- and close-ended questions. The completed questionnaires were collated, data extracted and cleansed (to check for data errors, outliers and missing values), thereafter processed in IBM SPSS statistical package (see [19]); while qualitative data was subjected to thematic analysis (see [20-22]).

\subsection{Design / Authoring of Proposed ePM-based Module}

The proposed intelligent learning module (iLM) is designed, configured and constructed using an existing authoring tool (i.e. ILABS-Intelligent Learning Activity Builder System, details to be presented in another research work - see fig. 2 above). The ePM concept is operationalized in the iLM via a virtual device within a numerical problem-solving context. That is, iLM is deplorable within the procedural aspects of applied numeric disciplines such as electrical engineering, physics, operation research, etc. Thus, several learning modules can be constructed. Each of the constructed modules can cover a specific topic within a target numeric discipline; 
the target topic thus constitutes the learning module's domain knowledge. Also, each learning module consists of meta-models (i.e. teaching, domain knowledge, generic-problem and user interface meta-models) and a virtual calculator, all constructed and integrated using the earlier mentioned authoring tool. However, a collection of learning modules constructed (covering varying topics), using the authoring tool, can be deposited/uploaded into a chosen repository from where they can be downloaded/lunched through a learning management system.

The iLM's teaching meta-model supports two tutoring routes / strategies (i.e. ePM and Model-Tracing routes) and one non-tutoring route. Note, the implementation of ePM strategy is enabled through human-computer interaction via a virtual calculator that is built into the iLM. The latter strategy thus aims duo purposes: [i] to interactively track the cognitive activities of learners during procedural problem-solving sessions; [ii] to enhance generation of step-wise feedbacks by comparing each cognitive node/step with that of a domain expert; it thus attempts to address target node/step-level problem-solving task. Hence, ePM is regarded as a step-wise tracking learning process strategy.

On the other hand, the alternative tutoring route-the model-tracing (MT) strategy-requires no virtual calculator to function, but provides goal-oriented feedbacks. This MT tutoring mode compares learners' solution at the end of a target problem-solving goal with that of the domain expert. Thus, the reason the latter mode is referred to as goal-level tracking learning process strategy. Summarily, the two teaching strategies compare learners' solution with domain expert's version, but at different solution levels (node/step-also referred to as sub-goal-and goal levels) respectively. Also, each of the mentioned strategies generates appropriate feedback(s) at related operational level—node or goal level (see types of feedback in [23]).

For illustration and evaluation of the ePM strategy, as well as alternative strategy (i.e. MT), a prototype intelligent learning module (iLM) was constructed/generated from an authoring tool (i.e. ILABS) as earlier mentioned. Fig. 2 above shows a screenshot of the sample learning module with the authoring tool development environment at the background. Thus, this research paper demonstrates/examines the ePM strategy through the configuration/generation of a module to teach the concepts of marginal costing. Marginal costing is a numerical domain/subject; problems within this domain knowledge-by nature - are procedural to solve in that its concepts are derivable from and/or interlinked by rules (see fig. 3 below and appendix A for definition of the figure's domain variables/rules).

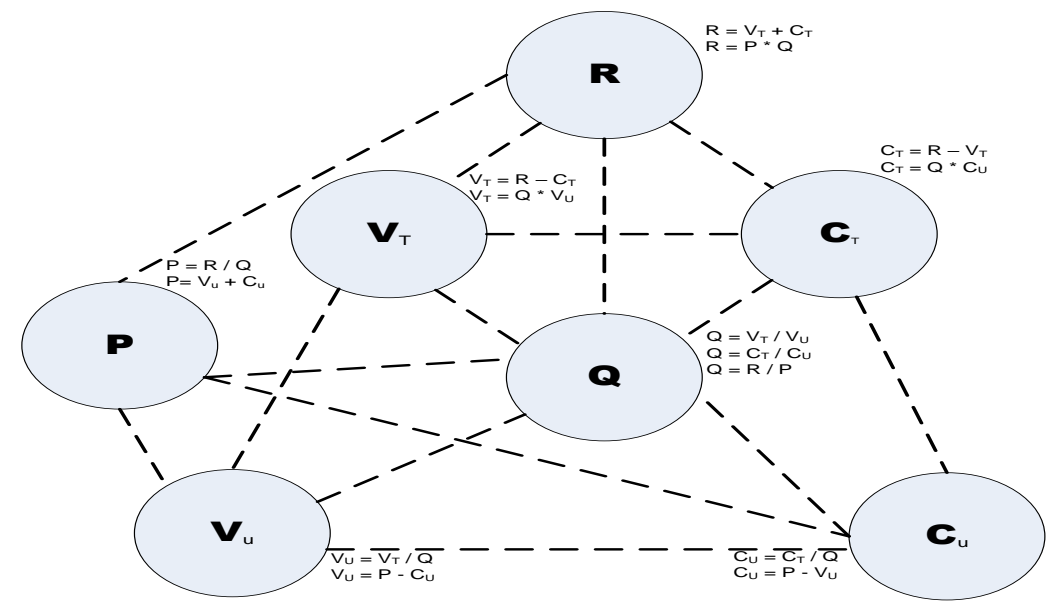

Fig. 3: Interlinked rules of marginal costing domain (Source: adopted from [24])

The learning module, deployed outside the authoring tool, is shown in fig. 4 below and consists of five (5) panels. It presents a panel for buttons (e.g. the close button); a panel that host target domain concepts-these concepts are represented by text boxes and connected to each other by built-in background rules; a virtual calculator panel; an interactive message panel where feedbacks generated in the course of learning are displayed; and lastly, a learning mode directives' panel—where actions to be undertaken during learning process are displayed. 
Operationally, the virtual calculator can be switched on or off subject to learner's choice of tutoring route/strategy. When the calculator is on, ePM is automatically active, thus enforces learning of concepts via the calculator. As a result, learners' step-wise movement between calculator and concepts' panel—that is, actions of

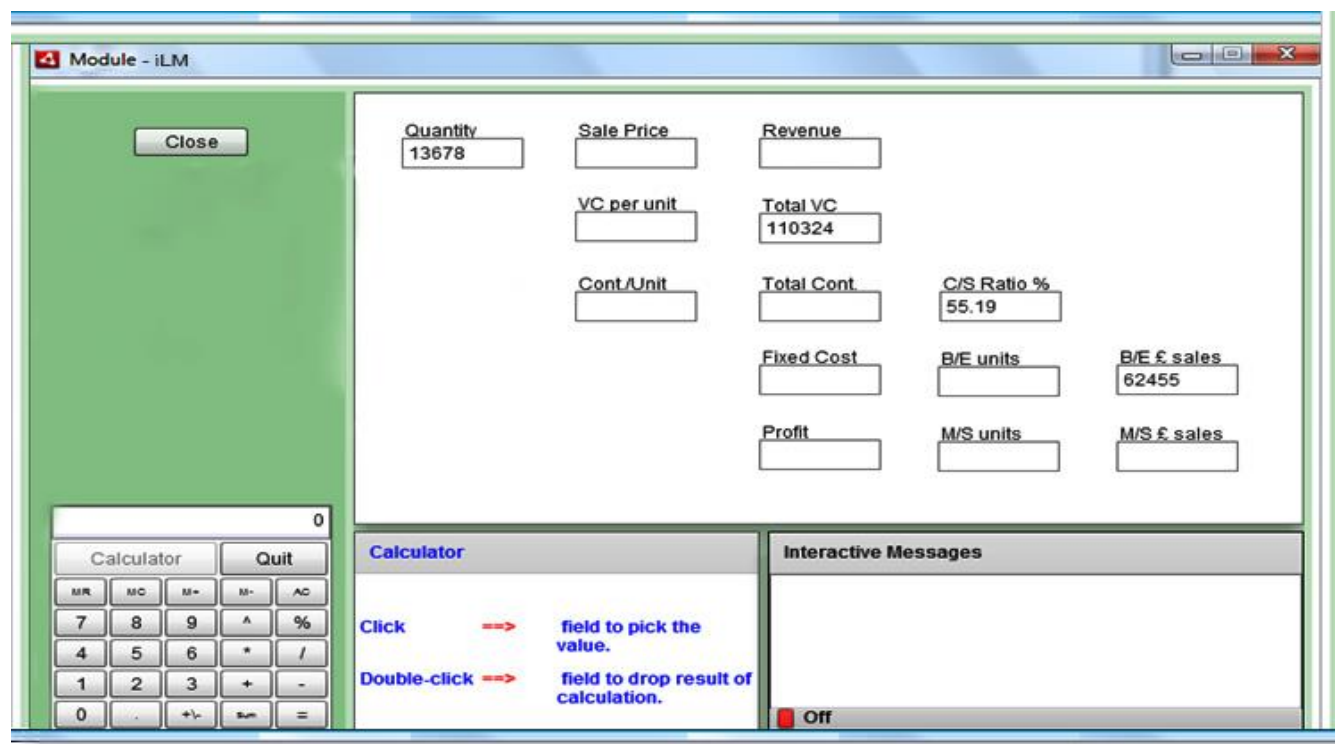

Fig. 4: The prototype intelligent learning module

picking and dropping values from/to text boxes and calculator-are all monitored and compared with expert domain solution steps. Any wrong step or move is immediately alerted with the generation and display of relevant feedback(s) on the interactive message panel. As earlier mentioned, this approach is known as step/node-oriented learning strategy which represents the implementation of the concept of interface-driven ePM.

Alternatively, the MT route/strategy is switched on once the virtual calculator is turn-off. The off-state thus compels users to learn domain concepts without using the learning module's calculator. The implication is that feedback is only provided with respect to the correctness or misconception of the domain concepts at the goal point or result level. Hence, error committed at any of the steps constituting the solution process cannot be detected. This approach or route is therefore known as goal-oriented or the model-tracing strategy as earlier discussed above.

However, a learner that chooses not to be guided or provided feedbacks during a learning process can turn-off the interactive message panel. By such decision or action, a learner can take any of the available learning routes but feedback won't be generated / displayed. Consequently, such a learner will be required to figure out issues/misconceptions that arise during the learning process. The on/off button can be found at the bottom of the interactive message panel of the learning module.

\section{RESULTS / ANALYSIS}

In this section, the result of the evaluation of the interface-driven ePM-based intelligent learning module in two education settings/institutions is presented. Table 1 below present the statistical distribution of three (300) hundred volunteer participants across the two institutions. These are second year students offering marginal costing as part of their degree curriculum.

Table 1: Statistics of Participants

\begin{tabular}{|l|l|l|}
\hline \multicolumn{2}{|l|}{ Study Institutions } & Questionnaire \\
\hline Study & Institution A & 151 \\
\hline & Institution B & 149 \\
\hline Total & & $\mathbf{3 0 0}$ \\
\hline
\end{tabular}


Based on the statistics in table 1 above, responses to both close-ended and open-ended questions were analysed with respect to two research propositions stated thus:

i. Learner's cognitive process can be made visible to domain expert (when learning is done through interaction with a virtual device).

ii. Cognitive visibility can aid the generation of timely and relevant diagnostic feedback.

Note that the first proposition provides answer to research objective (i), while the second proposition relates to objective (ii) as stated in section 1 of this paper.

\subsection{Quantitative Analysis}

This sub-section provides analysis of empirical data from close-ended questions that relate to the two research propositions only.

Proposition i: Learner's cognitive process can be made visible to domain expert (when learning is done through interaction with a virtual device).

This proposition aims to determine the perception/reaction of research subjects to ability of the learning module to track the cognitive process of the learners during learning process. To address this proposition, data related to two scales - Cognitive Process Visibility scale (COPVSB) and Conversation aids Cognitive Visibility (CACVSB) - in the deployed questionnaire were statistically analysed. The former scale determines the implementation of cognitive process visibility in the module. The latter deals with the operationalization of conversation via a virtual device (i.e. calculator) in order to facilitate accomplishment of the former construct. Theoretically, it is assumed that if a learner engaged in conversation with a domain expert, the thinking process of the learner could be revealed to the domain expert; hence, the scale CACVSB. Note that conversation conceived and implemented herein, means interactive conversation via a virtual device, not voice.

Tables $2 \mathrm{a}, 2 \mathrm{~b}$ and 3 below present the statistical analyses of responses to the close-ended questions with respect to the two referred scales. Tables $2 \mathrm{a}$ and $2 \mathrm{~b}$ represent the computation of one-sample statistics for scales COPVSB and CACVSB respectively, as well as their respective items. Table 3 represents the results of one sample t-test for COPVSB and CACVSB scales and their respective items.

Table 2a: One-Sample Statistics for COPVSB scale

\begin{tabular}{|l|l|l|l|l|}
\hline & N & Mean & Std. Deviation & Std. Error Mean \\
\hline Cognitive Process Visibility scale & $\mathbf{3 0 0}$ & $\mathbf{3 . 7 2 7 1 4}$ & $\mathbf{6 4 5 0 7 3}$ & $\mathbf{0 3 7 2 4 3}$ \\
\hline copvsb1 & 300 & 3.74 & .935 & .054 \\
\hline copvsb2 & 300 & 3.75 & .892 & 051 \\
\hline copvsb3 & 300 & 3.72 & .947 & .055 \\
\hline copvsb4 & 300 & 3.69 & .951 & .055 \\
\hline copvsb5 & 300 & 3.66 & .980 & .057 \\
\hline copvsb6 & 300 & 3.70 & 1.024 & .059 \\
\hline Copvsb7 & 300 & 3.83 & .879 & .051 \\
\hline
\end{tabular}

Table 2b: One-Sample Statistics for CACVSB scale

\begin{tabular}{|c|c|c|c|c|c|}
\hline & & $\mathbf{N}$ & Mean & Std. Deviation & Std. Error Mean \\
\hline $\begin{array}{l}\text { Conversation } \\
\text { Visibility scale }\end{array}$ & Cognitive & 300 & 3.89500 & .717536 & .041427 \\
\hline cacvsb1 & & 300 & 3.89 & .802 & .046 \\
\hline cacvsb2 & & 300 & 3.90 & 889 & .051 \\
\hline
\end{tabular}

For the purpose of analysis and to determine the direction of subjects' reaction/perception to the scales/items in the questionnaire, a benchmark value of 3.0 was adopted. Note that the value of each scale/scale items ranges from 5(strongly agree) to 1 (strongly disagree), thus the adopted benchmark value of 3.0 represents the mid-point 
which stands for neither agree nor disagree. This stance was taken since there is no previous learning module to compare with; also, this position/approach aligns with previous work in the literature (see [25]).

With respect to the one-sample statistics in tables $2 \mathrm{a}$ and $2 \mathrm{~b}$ above, the overall reaction mean scores for COPVSB and CACVSB scales are above the research's benchmark (3.0); that is, COPVSB - (M=3.73 approx., $\mathrm{SD}=0.645$ approx.), and CACVSB - (3.90 approx., $\mathrm{SD}=0.718$ approx.). By implication, it could be concluded that at both scales and items levels, respondents agree that the module makes visible or tracks learners' cognitive activities. Also, it could be stated that conversation between learners and the module (via the virtual interface device i.e. calculator) was instrumental to the visibility of learners' cognitive visibility.

Also, tables $2 \mathrm{a}$ and $2 \mathrm{~b}$ above shows that the mean values of individual scale's items align with their scale's positions since these values were above the benchmark value of 3.0. However, in scale COPVSB, item copvsb7- "responses from the iLM were relevant to my problem solving steps"-has highest mean score $(\mathrm{M}=3.81 ; \mathrm{SD}=0.879)$, while item copvsb5-“iLM responses show it accurately identified my thinking process" - has the lowest mean score $(\mathrm{M}=3.66, \mathrm{SD}=0.980)$. The latter scale item thus provides insight into the limitation or imperfection of a technology-based learning module due to its inability to capture the totality of the thinking process as evidence by the mean value of item copvsb5. Even if the interface-driven ePM module performed at optimal level, outcome of the evaluation shows that it is impossible to monitor close to $100 \%$ the problem-solving process of learners as voiced in response to scale item copvsb5.

Further to the foregoing, a t-test was undertaken to validate the above outcome/claim. The t-test result presented in table 3 below indicates significant differences between the two scales measured and the benchmark, thus:

- COPVSB: $\mathrm{t}(299)=19.524, \mathrm{p}=0.0$, and the magnitude of the difference $($ mean difference $=0.727$ at $95 \%$ CI: 0.654 to 0.800 ) was large (eta squared $=0.56$ );

- CACVSB: $\mathrm{t}(299)=21.604, \mathrm{p}=0.0$, and the magnitude of the difference (mean difference $=0.895$ at $95 \%$ CI:0.813 to 0.977 ) was large (eta squared $=0.61$ ).

Also, the scales' items show significant difference from the benchmark. However, as shown in the one-sample statistics table 2a above, item "copvsb5" "iLM responses show it accurately identified my thinking process", equally has the lowest mean score in the t-test; hence, this confirm the conclusion reached:

- COPVSB5: $\mathrm{t}(299)=11.662, \mathrm{p}=0.0$ (at alpha level 0.05), and the magnitude of the difference (mean difference $=0.660$ at $95 \%$ CI: 0.55 to 0.77 ) was large (eta squared $=0.31$ ). This result thus indicates that the interface-based ePM module enhanced cognitive visibility.

Even though the foregoing results represent the general perception of evaluators, it is unknown whether some factors (such as demographic characteristics, IT experience, etc.) influenced the recorded perceptions of research subjects. This is a possible future research issue that can be investigated.

\section{Proposition ii: Cognitive visibility can aid the generation of relevant and timely diagnostic feedback.}

This proposition aims to ascertain the perception/reaction of research subjects to the extent the learning module generates timely and relevant diagnostic feedbacks, in relation to the visibility of the cognitive process of learners. The proposition, therefore, attempts to establish linkage between cognitive visibility and two constructs-time (TIMFBK) and relevance of the diagnostic feedback (RELFBK) generated.

Preliminary analysis indicates that scales TIMFBK and RELFBK are not normally distributed. This necessitates the inspection of the mean and median values of both scales, in relation to the benchmark (3.0). Outcome of the inspection, however, indicates that the mean values for both scales are higher than their corresponding median, thus: timely feedback, TIMFBK $(\mathrm{M}=3.628$, Median=3.500, $\mathrm{SD}=0.691)$; and relevant feedback, RELFBK $(\mathrm{M}=3.874$, Median=3.833, $\mathrm{SD}=0.552)$. As well, the mean and median values for both scales are higher than the benchmark (3.0) - see table $4 \mathrm{a}$ and $4 \mathrm{~b}$ below for details. Thus, at scale level analysis, the foregoing outcomes suggest that subjects concur to constructs investigated.

Also, at scales' items level (see table $4 \mathrm{a}$ and $4 \mathrm{~b}$ above), mean values for the two scales' items were above the benchmark. This therefore suggests that subjects accede to the constructs measured. 
Table 3: One-Sample t- Test for COPVSB and CACVSB scales

\begin{tabular}{|c|c|c|c|c|c|c|}
\hline & & \multicolumn{5}{|c|}{ Test Value $=3.0($ benchmark $)$} \\
\hline & & \multirow[b]{2}{*}{ Df } & \multirow{2}{*}{$\begin{array}{l}\text { Sig. } \\
(2 \text {-tailed })\end{array}$} & \multirow{2}{*}{$\begin{array}{l}\text { Mean } \\
\text { Difference }\end{array}$} & \multicolumn{2}{|c|}{$\begin{array}{l}95 \% \text { Confidence } \\
\text { Interval of the } \\
\text { Difference }\end{array}$} \\
\hline & t & & & & Lower & Upper \\
\hline $\begin{array}{l}\text { Cognitive Process Visibility } \\
(\mathrm{COPVSB})\end{array}$ & sub-scale 19.524 & 299 & 000 & .727143 & .65385 & 80044 \\
\hline copvsbl & 13.774 & 299 & .000 & .743 & 64 & 85 \\
\hline copvsb2 & 14.633 & 299 & .000 & .753 & 65 & 85 \\
\hline copvsb3 & 13.232 & 299 & .000 & .723 & 62 & 83 \\
\hline copvsb4 & 12.503 & 299 & .000 & .687 & .58 & .79 \\
\hline copvsb5 & 11.662 & 299 & .000 & .660 & .55 & 77 \\
\hline copvsb6 & 11.786 & 299 & .000 & .697 & .58 & 81 \\
\hline copvsb7 & 16.290 & 299 & .000 & 827 & .73 & .93 \\
\hline $\begin{array}{l}\text { Conversation Aids Cognitive } \\
\text { sub-scale (CACVSB) }\end{array}$ & Visibility 21.604 & 299 & 000 & 895000 & 81347 & 97653 \\
\hline cacvsbl & 19.158 & 299 & .000 & .887 & 80 & .98 \\
\hline cacvsb2 & 17.604 & 299 & .000 & .903 & 80 & 1.00 \\
\hline
\end{tabular}

In spite of the above findings, further investigation with one-sample t-test was undertaken. A benchmark of 3.0 was set as basis for comparison in this analysis. The t-test result is presented in table 5 below. The result indicates statistically-significant differences, at both the scale and items levels, between the mean scores of scales (i.e. TIMFBK and RELFBK) and their items under consideration and the benchmark (3.0). The magnitudes of the differences (i.e. the computed eta-squared) for the aforementioned scales are given below:

- Timely feedback (TIMFBK) - $\mathrm{t}(299)=15.747, \mathrm{p}=0.0$; the magnitude of the difference (mean difference $=0.628$, 95\% CI: 0.550 to 0.707 ) was very large (eta squared=0.45); and

- Relevant feedback (RELFBK) - $\mathrm{t} \quad(298)=27.369, \mathrm{p}=0.0$; the magnitude of the difference (mean difference $=0.874,95 \% \mathrm{CI}: 0.811$ to 0.937 ) was very large (eta squared $=0.72$ ).

From the above analysis, users that learn via the virtual device appear to assent to both constructs. This implies that the operation of cognitive visibility strategy via the on-module virtual device aids the generation of timely and relevant feedback.

Table 4a: One-Sample Statistics for TIMFBK scale

\begin{tabular}{|c|c|c|c|c|}
\hline & $\mathbf{N}$ & Mean & Std. Deviation & Std. Error Mean \\
\hline Timely Feedback scale & 300 & 3.62833 & 691102 & 039901 \\
\hline timfbk1 & 300 & 3.89 & .847 & .049 \\
\hline timfbk2rvs & 300 & 3.36 & .963 & .056 \\
\hline
\end{tabular}

Table 4b: One-Sample Statistics for RELFBK scale

\begin{tabular}{|l|l|l|l|l|} 
& N & Mean & Std. Deviation & \multicolumn{1}{c|}{ Std. Error Mean } \\
\hline Relevant Feedback scale & $\mathbf{2 9 9}$ & $\mathbf{3 . 8 7 4 0 2}$ & $\mathbf{5 5 2 1 9 5}$ & $\mathbf{0 3 1 9 3 4}$ \\
\hline relfbk1 & 300 & 4.11 & .705 & .041 \\
\hline relfbk2 & 300 & 3.79 & .848 & .049 \\
\hline relfbk3 & 299 & 4.06 & .764 & .044 \\
\hline relfbk5 & 299 & 3.60 & .894 & .052 \\
\hline relfbk6 & 299 & 3.61 & 1.022 & .059 \\
\hline
\end{tabular}


Table 5: One-Sample Test for TIMFBK and RELFBK scales

\begin{tabular}{|c|c|c|c|c|c|c|}
\hline & \multicolumn{6}{|c|}{ Test Value $=3.0$ (benchmark value) } \\
\hline & \multirow[b]{2}{*}{$\mathrm{T}$} & \multirow[b]{2}{*}{$\mathrm{df}$} & \multirow{2}{*}{$\begin{array}{l}\text { Sig. } \\
\text { (2-tailed) }\end{array}$} & \multirow[b]{2}{*}{ Mean Difference } & \multicolumn{2}{|c|}{$\begin{array}{l}95 \% \text { Confidence Interval of } \\
\text { the Difference }\end{array}$} \\
\hline & & & & & Lower & Upper \\
\hline Timely Feedback scale & 15.747 & 299 & 000 & .628333 & 54981 & 70686 \\
\hline timfbk1 & 18.265 & 299 & .000 & .893 & 80 & 99 \\
\hline timfbk2rvs & 6.477 & 299 & 000 & .360 & .25 & .47 \\
\hline $\begin{array}{l}\text { Relevant } \\
\text { scale }\end{array}$ & 27.369 & 298 & 000 & 874025 & 81118 & 93687 \\
\hline relfbk1 & 27.191 & 299 & .000 & 1.107 & 1.03 & 1.19 \\
\hline relfbk2 & 16.197 & 299 & .000 & .793 & .70 & 89 \\
\hline relfbk3 & 24.082 & 298 & .000 & 1.064 & .98 & 1.15 \\
\hline relfbk5 & 11.518 & 298 & .000 & .595 & .49 & .70 \\
\hline relfbk6 & 10.239 & 298 & .000 & .605 & .49 & .72 \\
\hline relfbk7 & 22.573 & 298 & .000 & 1.080 & .99 & 1.17 \\
\hline
\end{tabular}

\subsection{Qualitative Analysis}

Current sub-section reports the analysis of responses to open-ended items of the research instrument. Note that only few subjects attended to this section of the questionnaire. Notwithstanding, this paper did not ignore their responses to forestall bias to the presentation herein. Also, some of the observations during the evaluation session are equally reported. As presented below, the responses reflect satisfaction with the learning module. Also, comments on the weakness of the learning module are stated. Both categories of responses provide insights into the perception of research subjects with respect to the implementation of ePM via an interface and its consequential effect on the generation of diagnostic feedbacks.

Stated below are some positive remarks from the completed questionnaires as they relate to issues addressed in this paper:

- Makes the user think of the problem, and stimulates the mind.

- Responding to each action to avoid misconception in the process.

- It had an alternative twist to learning, which may be beneficial for students who like alternative ways.

Also, remarks relating to weakness of the learning module and areas needing improvement are stated thus:

- $\quad$ Solving problem is just limited to certain level.

- Feedback should give formula immediately when wrong.

- The learning module can give audio feedbacks which will be more interactive.

From above qualitative analysis, research subjects' express satisfaction with the learning module. A few though indicates discomfort with the frequency of the feedbacks given. Occasionally, it was observed that some evaluators turn-off the process-monitoring learning route on grounds that the frequent feedbacks (i.e. immediate feedbacks) hinder their thought process. This latter category of evaluators prefers to explore the learning module, discover knowledge, instead of being guided. Thus, the action of the latter category contrast with comments of evaluators that prefer the immediate (or frequent) feedbacks, as earlier indicated.

\section{DISCUSSION}

As presented above, two research issues are investigated through the proposed interface-driven ePM approach that is demonstrated through the authoring of an intelligent learning module (iLM). The first, to track or make visible the cognitive process of learners during a learning process (termed cognitive visibility); while the second 
issue, is the utilisation of revealed cognitive process to aid generation of timely and relevant diagnostic feedbacks.

The philosophy that underpins this research is that if the cognitive process of learners could be made visible to domain expert, the iLM that consist of the domain expert can intelligently and adaptively respond through the generation of relevant diagnostic feedbacks that could support learners.

Accordingly, results in section 5.0 revealed certain positions with respect to above stated issues under investigation. Findings indicates that the interaction or conversation between users and the iLM via a virtual calculate actually revealed the cognitive process of learners. It thus confirms that the built-in ePM strategy, which implements the cognitive visibility concept, worked as intended in the designed learning module. The ePM strategy constitute one of the trio tutoring strategies (process monitoring, model tracing and non-tutoring routes) built into the learning system.

As earlier mentioned, the implementation of ePM enforces learning via a virtual calculator (an interface on the iLM; act as the conversation medium between a learner and the learning module), unlike the other two tutoring routes. Thus, ePM working behaviour ensures that the virtual calculator captures learning process in a step-wise pattern, mapping learning nodes as the learning progresses.

Although, the concept of cognitive visibility investigated in this research appears similar to closely-related concepts (e.g. cognitive mapping, student modelling, pattern or plan recognition - see [11], [26]) discussed in previous literatures, the interface-driven ePM design approach is uniquely different from earlier works. While earlier works investigated/implemented cognitive related issues with the utilisation of artificial intelligence techniques (such as Fuzzy Logic, Bayesian Networks, RNN etc.—see [10], [11], [13]), current work approached the concept from the stand point of human computer interaction and utilised a rule-based approach to interpret learners' inputs via a calculator interface. Hence, the reason the approach is termed interface-driven ePM approach.

On the other hand, research subjects opine that ePM impacted generation of diagnostic feedbacks. The empirical data suggests that ePM enables the provision of timely and relevant diagnostics feedbacks. However, feedbacks are provided as reward or guidance in response to users' misconceptions of concepts being learnt. Immediate feedbacks are noticeable when ePM route is turn-on, while delayed feedback is given when model-tracing route is activated. However, feedback is not generated when neither of the learning modes is activated. Also, it was observed that immediate feedbacks are generated at minute node- or step-levels of a task, while goal-level feedbacks (otherwise known as delayed feedbacks) are given on completion of a task. The research findings thus demonstrate that the module's built-in feedback generator relates feedbacks to problem states base on the learning route activated. Note that feedback generator is one of the features built into the learning module; it behaviours according to the learning route activated or turned-on. In addition, this research confirms a link between the ePM and feedback mechanisms; and that it worked as designed. It could therefore be concluded that the ePM that is instrumental to the actualisation of cognitive visibility or tracking of the cognitive process of learners actually enhanced the generation of timely and relevant diagnostic feedbacks.

Qualitative perspective (i.e. responses to the open-ended questions of the research instrument) indicates that cognitive visibility (i.e. the ePM) route is useful at the early stage of learning. This is due to display of prompt feedbacks. Thus, ePM is helpful in shaping learning at this stage. On the other hand, advance learners felt that frequent feedbacks interfere with smooth learning process; it prevents reflection whenever a misconception is noticed. This latter category of learners prefers to think through a problem, change their course of action/direction, instead of being guided or provided hints.

However, they are quick to note, that the learning module provides opportunity to switch off the ePM route that is responsible for the frequent feedbacks; turn-on either the delayed feedback option or no feedback route. These aforementioned provisions on the learning module thus take care of the learning needs of the advance learners.

\section{CONCLUSION}

In view of the above, this research proved that learners' cognitive process can be made visible or tracked through interactive learning conversations using ePM via an interface (i.e. a virtual calculator). That ePM enhances the generation of timely and relevant diagnostic feedbacks. Thus, the proposed interface-driven 
embedded process monitoring tracks cognitive process of learners with consequential impact on the generation of diagnostic feedbacks.

Through his research effort, current work provides a conceptual connection between ePM, cognitive visibility and feedback generation in a technology-based learning environment. Therefore, the conceptualisation and practical implementation of cognitive visibility via virtual calculator (i.e. an interface) is a unique/significant contribution. This work demonstrates an alternative implementation of intelligence in learning modules outside previous predominantly AI techniques research works. Also, this implementation of intelligence-through ePM over an interface-has an advantage over standard AI techniques because the latter (i.e.AI-based implementation) mainly rely on inference which may occasionally fails.

However, this research notes for future consideration, both the positive and negative impact of ePM on learners' cognitive process and feedback generation.

\section{ACKNOWLEDGMENTS}

This is an updated work of a research partly funded with the Teaching and Learning research grant from De Montfort University, UK, through the support of Ashok Patel, my research mentor; his support is acknowledged and appreciated.

\section{APPENDIX}

According to Patel and Kinshuk [24], figure 3 above describes partial networks of seven (7) out of 14 concepts/variables for solving problems in marginal costing. These networks are based on the following equations/rules:

$$
\begin{array}{llll}
\mathrm{R} & =\mathrm{Q} * \mathrm{P} & \Rightarrow & \text { Revenue }=\text { Quantity } * \text { Price } \\
\mathrm{VT} & =\mathrm{Q} * \mathrm{VU} & \Rightarrow & \text { Variable (total) cost }=\text { Quantity } * \text { Variable (unit) cost } \\
\mathrm{CT} & =\mathrm{Q} * \mathrm{CU} & \Rightarrow> & \text { Contribution (total) }=\text { Quantity } * \text { Contribution (unit) } \\
\mathrm{CT} & =\mathrm{R} * \mathrm{VT} & \Rightarrow & \text { Contribution (total) }=\text { Revenue } * \text { Variable (total) cost }
\end{array}
$$

Note, a unit or node can be an arithmetic operator (or any other operand e.g. “+”, “-“, “*”), a variable (regarded as a container that holds a value e.g. cost, sales, quantity) and a predefined or imported function(s).

All the nodes - variables, operands, functions - and the relationship (i.e. operators) that exist between them thus represents the domain knowledge (i.e. the learning content). Note that the domain knowledge domiciles in the domain module of ITS, one of the four models of an ITS.

\section{REFERENCES}

[1] K. Crockett, A. Latham, N. Whitton, "On predicting learning styles in conversational intelligent tutoring systems using fuzzy decision trees”. International Journal of Human-Computer Studies, Vol. 97, 2017, 98115 .

[2] L. Liu, W. Wu, J. Huang, "SARLR: Self-adaptive Recommendation of Learning Resources". Proceedings of $14^{\text {th }}$ International Conference, ITS 2018, Montreal, QC, Canada, June 11-15, 2018, 151-168.

[3] H. Mohamed, M. Lamia, "Implementing flipped classroom that used an intelligent tutoring system into learning process”. Computers \& Education, Vol. 124, No. 1, 2018, 62-76.

[4] K. Kularbphettong, P. Kedsiribut, P. Roonrakwit, "Developing an Adaptive Web-based Intelligent Tutoring System Using Mastery Learning Technique”. Procedia-Social and Behavioral Sciences, Vol. 191, No. 2, 2015, 686-691.

[5] Murray, T. An Overview of Intelligent Tutoring System Authoring Tools : Updated Analysis Of The State Of The Art. In: Murray, T., Blessing, S. and Ainsworth S. (Eds.). Authoring Tools for Advanced Technology Learning Environments, Kluwer Academic Publishers, 2003. 
[6] Nkambou, R., Bourdeau, J., Psyche, V. Building Intelligent Tutoring Systems: An Overview. In: Nkambou, R., Bourdeau, J. and Mizoguchi, R. (Eds.), Advances in Intelligent Tutoring Systems, Berlin Heidelberg: Springer-Verlag, 2010, 361-376.

[7] F. Bouchet, J. M. Harley, G. J. Trevors, R. Azevedo, "Clustering and profiling students according to their interactions with an intelligent tutoring system fostering self-regulated learning", J. Edu. Data Mining, Vol. 5, No. 1, 2013, 104-146.

[8] D. Hooshyar, R.B. Ahmad, M. Yousefi, F.D. Yusop, S.J. Horng, “A flowchart-based intelligent tutoring system for improving problem-solving skills of novice programmers". Journal of Computer Assisted Learning, Vol. 31, No. 4, 2015, 345-361.

[9] D. Hooshyar, R.B. Ahmad, S. Shamshirband, M. Yousefi, S.J. Horng, "A flowchart-based programming environment for improving problem solving skills of Cs minors in computer programming". The Asian International Journal of Life Sciences, Vol. 24, No. 2, 2015, 629-646.

[10] V. Chieu, V. Luengo, L. Vadcard, J. Tonetti, "Student Modeling in Orthopedic Surgery Training: Exploiting Symbiosis between Temporal Bayesian Networks and Fine-grained Didactic Analysis". International Journal of Artificial Intelligence in Education, Vol. 20, No. 3, 2010, 269-301.

[11] C. Piech, J. Bassen, J. Huang, S. Ganguli, M. Sahami, L.J. Guibas, J. Sohl-Dickstein, "Deep knowledge tracing". Advances in Neural Information Processing Systems, Montreal, Canada, December 7-12, 2015, 505-513.

[12] K. Ostrow, C. Donnelly, S. Adjei, N. Heffernan, "Improving student modeling through partial credit and problem difficulty”. Proceedings of the Second (2015) ACM Conference on Learning at Scale, Vancouver, Canada, March 14-15, 2015, 11-20.

[13] M. Eagle, A. Corbett, J. Stamper, B.M. McLaren, R. Baker, A. Wagner, A. Mitchell, "Predicting Individual Differences for Learner Modeling in Intelligent Tutors from Previous Learner Activities". Proceedings of the $24^{\text {th }}$ ACM International Conference on User Modeling Adaptation and Personalization, Halifax, Canada, July 13-16, 2016, 55-63.

[14] L. Han, “An Interdisciplinary Intelligent Teaching System Model Based on College Students' Cognitive Ability". 2018 International Conference on Virtual Reality and Intelligent Systems (ICVRIS), Hunan, China, August 10-11, 2018, 259-262.

[15] M. Waalkens, V. Aleven, N. Taatgen, "Does supporting multiple student strategies lead to greater learning and motivation? Investigating a source of complexity in the architecture of intelligent tutoring systems". Computers \& Education, Vol. 60, No. 1, 2013, 159-171.

[16] Gillham, B. Developing a Questionnaire. 2 ${ }^{\text {nd }}$ ed. London, UK: Bloomsbury Publishing Plc, 2008.

[17] L. Thabane, J. Ma, R. Chu, J. Cheng, A. Ismaila, L.P. Rios, R. Robson, et al., "A tutorial on pilot studies: the what, why and how". BMC medical research methodology, Vol. 10, No. 1, 2010, 1-10.

[18] S. Chatterjee, P. Datta, S. Datta, A. Ghosh, G. Sarker, R. Pal, R., “Assessment of psychosocial problems among HIV positive individuals attending in the Teaching Institutes of West Bengal and Bihar, India". Al Ameen Journal of Medical Sciences, Vol. 11, No. 3, 2018, 191-198.

[19] Pallant, J. SPSS Survival Manual: A Step by Step Guide to Data Analysis using IBM SPSS. 6th ed. England: Open University Press, McGraw-Hill House, 2016.

[20] P. Burnard, P. Gill, K. Stewart, E. Treasure, B. Chadwick, “Analysing and presenting qualitative data". British dental journal, Vol. 204, No. 8, 2008, 429-32. 
[21] R.J. Chenail, "Conducting Qualitative Data Analysis : Qualitative Data Analysis as a Metaphoric Process". The Qualitative Report, Vol. 17, No. 1, 2012, 248-253.

[22] Silverman, D. Interpreting qualitative data. $5^{\text {th }}$ ed. London: Sage Publications Ltd., 2015.

[23] K. VanLehn, "The behavior of tutoring systems". International Journal of Artificial Intelligence in Education, Vol. 16, No. 3, 2006, 227-265. IOS Press.

[24] A. Patel, Kinshuk, "Intelligent Tutoring Tools in a Computer-Integrated Learning Environment for Introductory Numeric Disciplines”. Innovations in Education and Teaching International, Vol. 34, No. 3, 1997, 200-207.

[25] A. Granic, "Experience with usability evaluation of e-learning systems". Universal Access in the Information Society, Vol. 7, No. 4, 2008, 209-221.

[26] K. Chrysafiadi, M. Virvou, "Student modeling approaches: A literature review for the last decade". Expert Systems with Applications, Vol. 40, No. 11, 2013, 4715-4717. Elsevier. 